\title{
Characterizing Definability of Second-Order Generalized Quantifiers ${ }^{\star}$
}

\author{
Juha Kontinen $^{1}$ and Jakub Szymanik ${ }^{2}$ \\ 1 Department of Mathematics and Statistics, University of Helsinki \\ juha.kontinen@helsinki.fi \\ 2 Institute of Artificial Intelligence, University of Groningen \\ jakub.szymanik@gmail.com
}

\begin{abstract}
We study definability of second-order generalized quantifiers. We show that the question whether a second-order generalized quantifier $\mathcal{Q}_{1}$ is definable in terms of another quantifier $\mathcal{Q}_{2}$, the base logic being monadic second-order logic, reduces to the question if a quantifier $\mathcal{Q}_{1}^{\star}$ is definable in $\operatorname{FO}\left(\mathcal{Q}_{2}^{\star},<,+, \times\right)$ for certain first-order quantifiers $\mathcal{Q}_{1}^{\star}$ and $\mathcal{Q}_{2}^{\star}$. We use our characterization to show new definability and nondefinability results for second-order generalized quantifiers. In particular, we show that the monadic second-order majority quantifier Most ${ }^{1}$ is not definable in second-order logic.
\end{abstract}

\section{Introduction}

The notion of generalized quantifier goes back to Mostowski [1] and Lindström [2]. Generalized quantifiers were first mainly studied in the framework of model theory. The study of generalized quantifiers extended to the context of finite model theory via applications to descriptive complexity theory. We refer to [3] and [4] for surveys of first-order generalized quantifiers in finite model theory. Generalized quantifiers have been also extensively studied in the formal semantics of natural language (see [5] for a survey).

The study of second-order generalized quantifiers is a relatively new and unexplored area in finite model theory. On the other hand, second-order logic (SO) and its many fragments have been studied extensively starting from Fagin's characterization of NP in terms of existential second-order logic [6]. Second-order generalized quantifiers were first studied in the context of finite structures by Burtschick and Vollmer [7]. Shortly after, Andersson [8] studied the expressive power of families of second-order generalized quantifiers determined by the syntactic types of quantifiers. In $[9,10,11]$ Kontinen studied definability questions of second-order generalized quantifiers. In the case of first-order quantifiers, definability of a quantifier $Q$ in a logic $\mathcal{L}$ means that the class of structures, used to interpret $Q$, is axiomatizable in $\mathcal{L}$. In the second-order case, the analogous

\footnotetext{
* The first author was supported by grant 127661 of the Academy of Finland. The
} second author was supported by Vici grant NWO-277-80-001. 
concept of definability was formulated in $[9,10]$. In this article, we give a computationally motivated characterization for the notion of definability of second-order generalized quantifiers.

Burtschick and Vollmer [7] noticed that second-order generalized quantifiers can be used to logically characterize complexity classes defined in terms of socalled Leaf Languages. The leaf languages approach in computational complexity theory, introduced by Bovet, Crescenzi, and Silvestri [12], is a unifying approach to define complexity classes. The central idea behind this approach is to generalize the conditions under which, e.g., a Turing machine or an automaton accepts its input. Many complexity classes can be defined in this context in terms of suitable leaf languages. On the other hand, a complexity class defined in terms of a leaf language $B$ can be under certain conditions characterized logically in terms of a logic of the form:

$$
\mathcal{Q}_{B} \text { FO, }
$$

where $\mathcal{Q}_{B}$ is a second-order generalized quantifier corresponding to the language $B$. In the context of leaf languages, polynomial time non-deterministic Turing machines can be sometimes replaced by non-deterministic finite automata (socalled finite leaf automata) without a significant increase in complexity [13]. Galota and Vollmer [14] showed that complexity classes defined in terms of finite leaf automata can be logically characterized in terms of monadic secondorder generalized quantifiers. This result nicely extends the well known $[15,16,17]$ characterization of regular languages in terms of monadic second-order logic (MSO).

The definability theory of second-order generalized quantifiers has some similarities and differences compared to that of first-order generalized quantifiers. For example, it was observed in [9] that the binary second-order existential quantifier cannot be defined in terms of any monadic second-order generalized quantifiers. This result is in contrast with fact (a corollary of a result of Andersson [8]) that all classes of finite first-order structures are already definable in terms of monadic second-order generalized quantifiers. In this paper we prove a general result characterizing the question when a quantifier $\mathcal{Q}$ is definable in $\operatorname{MSO}\left(\mathcal{Q}^{\prime},+\right)$, where + denotes the built-in addition relation. We assume the built-in addition in order to unleash the expressive power embodied by MSO. Recall that, while MSO corresponds to regular languages over strings, $\mathrm{MSO}(+)$ corresponds to the linear fragment of the polynomial hierarchy (LINH) on strings [18]. It is possible to formulate our characterization also in the case where the base logic is full second-order logic instead of $\mathrm{MSO}(+)$.

Our characterization is based on a logical formalization of an idea of Torán [19]. Torán studied oracle separations in the counting hierarchy and noticed that there is essentially no difference between an oracle Turing machine writing an oracle query on its query tape and a logarithmic time Turing machine writing an address on its random access tape. He used this analogy to show that an oracle separation result for classes in the polynomial counting hierarchy implies a real separation for the corresponding classes in the logarithmic counting hierarchy LINCH (equivalently in DLOGTIME-uniform $\mathrm{TC}^{0}$ ). We show that a second- 
order generalized quantifier $\mathcal{Q}_{1}$ is definable in the $\operatorname{logic} \operatorname{MSO}\left(\mathcal{Q}_{2},+\right)$ iff for certain first-order encodings $\mathcal{Q}_{i}^{\star}$ of $\mathcal{Q}_{i}, \mathcal{Q}_{1}^{\star}$ is definable in $\mathrm{FO}\left(\mathcal{Q}_{2}^{\star},+, \times\right)$. It is worth noting that the latter condition implies that $\mathcal{Q}_{1}^{\star}$ is $\mathrm{AC}^{0}$ (Turing) reducible to $\mathcal{Q}_{2}^{\star}$. We use our characterization to show new definability and non-definability results for second-order generalized quantifiers. In particular, we show that the monadic second-order majority quantifier Most ${ }^{1}$ is not definable in second-order logic. This aswers the question left open in [20] (see also [21]), where second-order generalized quantifiers were used to model collective quantification in natural language. For the sake of brevity, we do not discuss the role and use of quantifiers in formal semantics in this paper.

\section{Preliminaries}

In this article all structures are assumed to be finite. The universe of a structure $\mathfrak{A}$ is denoted by $A$. Without loss of generality, we may assume that $A$ is always of the form $\{0, \ldots, m\}$ for some $m \in \mathbb{N}$. For a logic $\mathcal{L}$, the set of $\tau$-formulas of $\mathcal{L}$ is denoted by $\mathcal{L}[\tau]$. If $\phi$ is a $\tau$-sentence, then the class of $\tau$-models of $\phi$ is denoted by $\operatorname{Mod}(\phi)$. A class $K$ of $\tau$-models is said to be axiomatizable in a logic $\mathcal{L}$, if $K=\operatorname{Mod}(\phi)$ for some sentence $\phi \in \mathcal{L}[\tau]$. For logics $\mathcal{L}$ and $\mathcal{L}^{\prime}$, we write $\mathcal{L} \leq \mathcal{L}^{\prime}$, if for every $\tau$ and every sentence $\phi \in \mathcal{L}[\tau]$ there is a sentence $\psi \in \mathcal{L}^{\prime}[\tau]$ such that $\operatorname{Mod}(\phi)=\operatorname{Mod}(\psi)$. The set of natural numbers is denoted by $\mathbb{N}$ and $\mathbb{N}^{*}$ denotes the set $\mathbb{N} \backslash\{0\}$.

Sometimes we assume that our structures (and logics) are equipped with auxiliary built-in relations. In addition to the built-in ordering $<$, which is interpreted naturally, we also use the ternary relations + and $\times$. The relations + and $\times$ are defined as

$$
\begin{aligned}
& +(i, j, k) \Leftrightarrow i+j=k, \\
& \times(i, j, k) \Leftrightarrow i \times j=k .
\end{aligned}
$$

The relation BIT is a further important relation which is defined by: $\operatorname{BIT}(a, j)$ holds iff the bit of order $2^{j}$ is 1 in the binary representation $\operatorname{bin}(a)$ of $a$. The presence of built-in relations is signalled, e.g., by the notation $\mathrm{FO}(<)$. It is well known that $\mathrm{FO}(<,+, \times) \equiv \mathrm{FO}(<, \mathrm{BIT})$ (see [22]). Note that $<$ is easily definable in $\mathrm{FO}(+)$ and hence, in the precence of + , we sometimes do not mention < explicitely.

We assume that the reader is familiar with the basics of computational complexity theory. Below, we recall certain results from descriptive complexity theory. It is instructive to note that many of the logics considered in this article correspond to interesting complexity classes. We mention first the logic $\mathrm{FO}(<,+, \times)$ which corresponds exactly to the so-called logarithmic hierarchy (LH). This class is the logarithmic analogue of the polynomial hierarchy $(\mathrm{PH})$, corresponding to SO [23], defined in terms of alternating Turing machines (ATM) running in polynomial time with $O(1)$ alternations. In between $\mathrm{LH}$ and $\mathrm{PH}$ we have the linear hierarchy (LINH) corresponding to the logic $\mathrm{MSO}(+)$ over strings [18]. 
In this article also majority quantifiers are dicussed and studied. It is wellknown that majority quantifiers can be used to logically characterize counting computations. The following counting hierarchies are relevant for this article: the logarithmic counting hierarchy (LCH), the linear counting hierarchy (LINCH), and the (polynomial) counting hierarchy $(\mathrm{CH})$ all of which can be defined, with analogous resource bounds as LH, LINH, and PH, in terms of so-called Threshold Turing machines [24]. On the logical side, majority quantifiers (defined in Section 2.1) can be used to provide logical counterparts for these classes: $\mathrm{FO}(\mathrm{M},+, \times) \equiv$ $\mathrm{LCH}[25], \mathrm{FO}\left(\mathrm{Most}^{1},<\right) \equiv \mathrm{LINCH}$ (over strings) [26], and $\mathrm{FO}\left(\mathrm{Most}^{k}\right)_{k \in \mathbb{N}^{*}} \equiv$ $\mathrm{CH}$ [27]. Furthermore, in circuit complexity, it is known that LH corresponds exactly to DLOGTIME-uniform $\mathrm{AC}^{0}$ and $\mathrm{LCH}$ to DLOGTIME-uniform $\mathrm{TC}^{0}$ [25]. Also, DLOGTIME-uniform $\mathrm{AC}^{0}[p]\left(\mathrm{AC}^{0}\right.$ with unbounded fan-in $\mathrm{MOD}_{p}$ gates) corresponds on the logical side to $\mathrm{FO}\left(\mathrm{D}_{k},+, \times\right)[25]$.

\subsection{Generalized quantifiers}

In this section we briefly recall some basics of generalized quantifiers.

Let $\tau=\left\{P_{1}, \ldots, P_{r}\right\}$ be a relational vocabulary, where $P_{i}$ is $l_{i}$-ary for $1 \leq i \leq$ $r$, and $Q$ a class of $\tau$-structures closed under isomorphisms. The class $Q$ gives rise to a generalized quantifier which we also denote by $Q$. The tuple $s=\left(l_{1}, \ldots, l_{r}\right)$ is the type of the quantifier $Q$.

Definition 1. The extension $\mathrm{FO}(Q)$ of first-order logic by a quantifier $Q$ is defined as follows:

1. The formula formation rules of $\mathrm{FO}$ are extended by the rule: if for $1 \leq i \leq r$, $\phi_{i}\left(\bar{x}_{i}\right)$ is a formula and $\bar{x}_{i}$ is an $l_{i}$-tuple of pairwise distinct variables then $Q \bar{x}_{1}, \ldots, \bar{x}_{r}\left(\phi_{1}\left(\bar{x}_{1}\right), \ldots, \phi_{r}\left(\bar{x}_{r}\right)\right)$ is a formula.

2. The satisfaction relation of $\mathrm{FO}$ is extended by the rule:

$$
\mathfrak{A} \models Q \bar{x}_{1}, \ldots, \bar{x}_{r}\left(\phi_{1}\left(\bar{x}_{1}\right), \ldots, \phi_{r}\left(\bar{x}_{r}\right)\right) \text { iff }\left(A, \phi_{1}^{\mathfrak{A}}, \ldots, \phi_{r}^{\mathfrak{A}}\right) \in Q,
$$

where $\phi_{i}^{\mathfrak{A}}=\left\{\bar{a} \in A^{l_{i}} \mid \mathfrak{A} \models \phi_{i}(\bar{a})\right\}$.

We say that a quantifier $Q$ is definable in a logic $\mathcal{L}$ if the class $Q$ is axiomatizable in $\mathcal{L}$. Note that $Q$ is trivially definable in $\operatorname{FO}(Q)$. If $\mathcal{L}$ has the substitution property and is closed under FO-operations, then definability of $Q$ in $\mathcal{L}$ implies that $\mathrm{FO}(Q) \leq \mathcal{L}$. So, among such logics, $\mathrm{FO}(Q)$ is the minimal logic in which $Q$ is definable.

Example 1. The following quantifiers will be discussed in the following sections. Suppose $S \subseteq \mathbb{N}$ and $k \in \mathbb{N}$.

$$
\begin{aligned}
\exists & =\{(A, P) \mid P \subseteq A \text { and } P \neq \emptyset\} \\
\mathrm{M} & =\{(A, P) \mid P \subseteq A \text { and }|P|>|A| / 2\} \\
Q_{S} & =\{(A, P) \mid P \subseteq A \text { and }|P| \in S\}
\end{aligned}
$$

If $S$ is of the form $\{k n \mid n \in \mathbb{N}\}$ for some $k \in \mathbb{N}$, we denote $Q_{S}$ by $\mathrm{D}_{k}$. 
We will also refer to the vectorizations of the quantifiers $\mathrm{D}_{k}$ and $\mathrm{M}$ later. The $n$th vectorization of $\mathrm{D}_{k}$ is the following quantifier

$$
\mathrm{D}_{k}^{n}=\left\{(A, P) \mid P \subseteq A^{n} \text { and }|P|=0 \bmod k\right\},
$$

and the $n$th vectorization of $\mathrm{M}$ is

$$
\mathrm{M}^{n}=\left\{(A, P) \mid P \subseteq A^{n} \text { and }|P|>\left|A^{n}\right| / 2\right\} .
$$

Let us then turn to second-order generalized quantifiers. Let $t=\left(s_{1}, \ldots, s_{w}\right)$, where $s_{i}=\left(l_{1}^{i}, \ldots, l_{r_{i}}^{i}\right)$ is a tuple of positive integers for $1 \leq i \leq w$. A secondorder structure of type $t$ is a structure of the form $\left(A, P_{1}, \ldots, P_{w}\right)$, where $P_{i} \subseteq$ $\mathcal{P}\left(A^{l_{1}^{i}}\right) \times \cdots \times \mathcal{P}\left(A^{l_{r_{i}}^{i}}\right)$.

Definition 2. A second-order generalized quantifier $\mathcal{Q}$ of type $t$ is a class of structures of type $t$ such that $\mathcal{Q}$ is closed under isomorphisms.

A quantifier $\mathcal{Q}$ is monadic if $l_{j}^{i}=1$ for all $1 \leq j \leq r_{i}$ and $1 \leq i \leq w$. Let us look at some examples of second-order generalized quantifiers.

Example 2. Suppose $S \subseteq \mathbb{N}$ and $k \in \mathbb{N}$.

$$
\begin{aligned}
\exists_{k}^{2} & =\left\{(A, P) \mid P \subseteq \mathcal{P}\left(A^{k}\right) \text { and } P \neq \emptyset\right\} \\
\text { Even }^{\prime} & =\{(A, P) \mid P \subseteq \mathcal{P}(A) \text { and }|P| \text { is even }\} \\
\text { Even }^{\prime} & =\{(A, P) \mid P \subseteq \mathcal{P}(A) \text { and } \forall X \in P(|X| \text { is even })\} \\
\text { Most }^{k} & =\left\{(A, P) \mid P \subseteq \mathcal{P}\left(A^{k}\right) \text { and }|P|>2^{|A|^{k}-1}\right\} \\
\mathcal{Q}_{S} & =\{(A, P) \mid P \subseteq \mathcal{P}(A) \text { and }|P| \in S\}
\end{aligned}
$$

Analogously to the first-order case, if $S$ is of the form $\{k n \mid n \in \mathbb{N}\}$ for some $k \in \mathbb{N}$, we denote $\mathcal{Q}_{S}$ by $\mathcal{D}_{k}$.

The first example is the familiar $k$-ary second-order existential quantifier. The quantifier Even says that a formula holds for an even number of subsets of the universe. On the other hand, the quantifier Even' says that all the subsets satisfying a formula have an even cardinality. The quantifier $\operatorname{Most}^{k}$ is the $k$-ary second-order version of $\mathrm{M}$ expressing that a formula holds for more than half of the $k$-ary relations.

Definition 3. The extension $\mathrm{FO}(\mathcal{Q})$ of $\mathrm{FO}$ by a quantifier $\mathcal{Q}$ is defined as follows:

1. The formula formation rules of $\mathrm{FO}$ are extended by the rule: if for $1 \leq i \leq w$, $\phi_{i}\left(\bar{X}_{i}\right)$ is a formula and $\bar{X}_{i}=\left(X_{1, i}, \ldots, X_{r_{i}, i}\right)$ is a tuple of pairwise distinct predicate variables such that the arity of $X_{j, i}$ is $l_{j}^{i}$ for $1 \leq j \leq r_{i}$, then

$$
\mathcal{Q} \bar{X}_{1}, \ldots, \bar{X}_{w}\left(\phi_{1}\left(\bar{X}_{1}\right), \ldots, \phi_{w}\left(\bar{X}_{w}\right)\right)
$$

is a formula.

2. Satisfaction relation of $\mathrm{FO}$ is extended by the rule:

$$
\mathfrak{A}=\mathcal{Q} \bar{X}_{1}, \ldots, \bar{X}_{w}\left(\phi_{1}, \ldots, \phi_{w}\right) \text { iff }\left(A, \phi_{1}^{\mathfrak{A}}, \ldots, \phi_{w}^{\mathfrak{A}}\right) \in \mathcal{Q},
$$

where $\phi_{i}^{\mathfrak{A}}=\left\{\bar{R} \in \mathcal{P}\left(A^{l_{1}^{i}}\right) \times \cdots \times \mathcal{P}\left(A^{l_{r_{i}}^{i}}\right) \mid \mathfrak{A} \models \phi_{i}(\bar{R})\right\}$. 


\subsection{Definability}

Recall that a first-order generalized quantifier $Q$ is definable in a logic $\mathcal{L}$ if the class $Q$ is axiomatizable in $\mathcal{L}$. This condition can be reformulated as follows assuming $\mathcal{L}$ has the substitution property:

Proposition 1 A first-order quantifier $Q$ is definable in a logic $\mathcal{L}$ if and only if $\mathcal{L} \equiv \mathcal{L}(Q)$

How do we formalize definability for second-order quantifiers? Intuitively, e.g., the monadic second-order existential quantifier $\exists_{1}^{2}$ is definable in a logic $\mathcal{L}$ if there is a uniform way to express

$$
\exists_{1}^{2} X \psi(X)
$$

for any formula $\psi(X)$ in the logic $\mathcal{L}$. Over a model $\mathfrak{A}, \psi(X)$ defines a collection of subsets

$$
\{C \subseteq A \mid \mathfrak{A} \models \psi(C)\},
$$

so the problem is to find a way to express the non-emptyness of this collection in a way which does not depend on the particular formula $\psi(X)$. This was formalized in [10] using second-order relations.

Definition 4. Let $\mathcal{L}$ be a logic, $t=\left(s_{1}, \ldots, s_{w}\right)$ a second-order type, and let $\mathcal{G}_{1}, \ldots, \mathcal{G}_{w}$ be first-order quantifier symbols of types $s_{1}, \ldots, s_{w}$.

1. The logic $\mathcal{L}\left(\mathcal{G}_{1}, \ldots, \mathcal{G}_{w}\right)$ is obtained by extending the syntax of $\mathcal{L}$ in terms of the quantifiers $\mathcal{G}_{1}, \ldots, \mathcal{G}_{w}$.

2. The models of $\mathcal{L}\left(\mathcal{G}_{1}, \ldots, \mathcal{G}_{w}\right)$ are of the form $\mathcal{A}=\left(\mathfrak{A}, G_{1}, \ldots, G_{w}\right)$, where $\mathfrak{A}$ is a first-order model and

$$
G_{i} \subseteq \mathcal{P}\left(A^{l_{1}^{i}}\right) \times \cdots \times \mathcal{P}\left(A^{l_{r_{i}}^{i}}\right) .
$$

3. The quantifiers $\mathcal{G}_{i}$ are interpreted using the relations $G_{i}$ :

$$
\mathcal{A} \models \mathcal{G}_{i} \bar{x}_{1}, \ldots, \bar{x}_{r_{i}}\left(\phi_{1}\left(\bar{x}_{1}\right), \ldots, \phi_{r_{i}}\left(\bar{x}_{r_{i}}\right)\right)
$$

$\operatorname{iff}\left(\phi_{1}^{\mathcal{A}}, \ldots, \phi_{r_{i}}^{\mathcal{A}}\right) \in G_{i}$.

Note that if $\phi \in \mathcal{L}\left(\mathcal{G}_{1}, \ldots, \mathcal{G}_{w}\right)$ is a sentence of vocabulary $\tau=\emptyset$. Then

$$
\operatorname{Mod}(\phi)=\left\{\left(A, G_{1}, \ldots, G_{w}\right) \mid\left(A, G_{1}, \ldots, G_{w}\right) \models \phi\right\}
$$

corresponds to a second-order generalized quantifier of type $t$. This observation can be used to formalize definability of second-order generalized quantifiers. Below, we assume that $\mathcal{L}$ is closed under substitution.

Definition 5. Let $\mathcal{Q}$ be a quantifier of type t. The quantifier $\mathcal{Q}$ is definable in a logic $\mathcal{L}$ if there is $\phi \in \mathcal{L}\left(\mathcal{G}_{1}, \ldots, \mathcal{G}_{w}\right)$ of vocabulary $\sigma=\emptyset$ such that for any t-structure $\left(A, G_{1}, \ldots, G_{w}\right)$,

$$
\left(A, G_{1}, \ldots, G_{w}\right) \models \phi \Leftrightarrow\left(A, G_{1}, \ldots, G_{w}\right) \in \mathcal{Q} .
$$


The following was shown in [10]:

Theorem 2. If $\mathcal{Q}$ is definable in $\mathcal{L}$ then $\mathcal{L} \equiv \mathcal{L}(\mathcal{Q})$.

The converse of Theorem 2 does not hold:

Theorem 3 ([10]). There is a quantifier $\mathcal{Q}$ of type $((1))$ which is not definable in $\mathrm{FO}$ and satisfies $\mathrm{FO} \equiv \mathrm{FO}(\mathcal{Q})$.

Definability questions of second-order quantifiers has been studied in $[10,11,27]$. We recall the following results.

Theorem 4 ([11]). Let $t$ be type and $\mathcal{B}_{t}$ the collection of all second-order quantifiers of types less than $t$. Then there is a quantifier $\mathcal{Q}$ of type $t$ such that $\mathcal{Q}$ is not definable in $\mathrm{SO}\left(\mathcal{B}_{t}\right)$.

Theorem 4 is proved with respect to a natural ordering of the types of secondorder generalized quantifiers. Theorem 4 is existential in nature and does not give us a concrete non-definable quantifier. It was observed in [9] that it is not so difficult to find concrete quantifiers which cannot be defined using any monadic quantifiers. Denote by $\mathbb{Q}$ the collection of all monadic second-order generalized quantifiers.

Theorem 5 ([9]). The quantifier $\exists_{2}^{2}$ is not definable in $\mathrm{FO}(\mathbb{Q})$.

It is worth noting that the logic $\mathrm{FO}(\mathbb{Q})$ is capable of defining all classes of firstorder structures (cf. Theorem 6.2 in [8]). Finally, we recall the following result about second-order majority quantifiers:

Theorem 6 ([27]). The quantifier $\exists_{k}^{2}$ is definable in $\mathrm{FO}\left(\mathrm{Most}^{k}\right)$.

It interesting to note that definability of Most ${ }^{1}$ in the logic SO would imply that $\mathrm{PH} \equiv \mathrm{CH}$ in computational complexity. This observation was discussed in [20]. In this paper we show that the quantifier Most ${ }^{1}$ is not definable in SO, but, analogously to Theorem 3, this non-definability result does not imply that $\mathrm{PH} \subsetneq \mathrm{CH}$.

\section{Characterizing definability}

The computational analogue of a first-order generalized quantifier is the notion of an oracle (see [22]). Let $Q$ be a quantifier of vocabulary $\tau$ and $\mathcal{L}$ a logic. The idea is that in $\mathcal{L}(Q)$ we can query "without a cost" if a definable $\tau$-structure $\mathfrak{A}$ is a member of the class $Q$. Recall that a second-order generalized quantifier $\mathcal{Q}$ of type $((1))$ is definable, e.g., in $\mathrm{SO}$ if there is a sentence $\phi \in \mathrm{SO}(\mathcal{G})$ such that for all second-order structures $(A, G)$ :

$$
(A, G) \models \phi \Leftrightarrow(A, G) \in \mathcal{Q} .
$$

It is not immediately clear how to view this notion in computational terms. The set $G$ corresponds to a local first-order quantifier and, if we treat $G$ as an oracle, 
then in (1) we are infact trying to define a property oracles. One way to proceed is to formalize definability of a quantifier $\mathcal{Q}$ in terms of oracle Turing machines that treat (a suitable initial segment) the oracle as part of the input. However, in this article we do not follow that idea as there is a more familiar route to take. An important observation here is that the set $G$ can be of exponential size compared to the domain $A$. This observation can be used to show that SO-definability of $\mathcal{Q}$ reduces to logarithmic time definability.

Our proof is based on a logical version of an idea of Torán [19] showing that there is essentially no difference between an oracle Turing machine writing an oracle query on its query tape, and a logarithmic time Turing machine writing an address on its random access tape. In other words, an oracle in the setting of polynomial time machines can be viewed as an input to a logarithmic time machine. We use a logical version of this idea: we show that $\mathrm{SO}$ and the relation $G$ in (1) can be replaced by FO and a unary relation $P$ by passing from $A$ to a domain of cardinality $2^{|A|}$.

In this section we mainly restrict attention to monadic second-order generalized quantifiers. We interpret definability of quantifiers in logics with built-in relations in the natural way. For example, a second-order quantifier $\mathcal{Q}$ of type $((1))$ is definable in $\operatorname{MSO}(+)$ if there is $\phi \in \operatorname{MSO}(\mathcal{G},+)$ such that for all structures $(A,+, G):(A,+, G) \models \phi \Leftrightarrow(A, G) \in \mathcal{Q}$. In particular, Theorem 2 can be proved analogously in this setting.

Next we define a first-order encoding of a second-order structure of type $t$, for a monadic $t$. We use the fact that there is a one-to-one correspondence between integers $m \in B=\left\{0, \ldots, 2^{n}-1\right\}$ and subsets of $A=\{0, \ldots, n-1\}$ seen as length- $n$ binary numbers. Therefore, relations of $A$ can be encoded in terms of tuples of elements of $B$ and, further, sets of relations of $A$ by relations of $B$.

Definition 6. Let $t=\left(s_{1}, \ldots, s_{w}\right)$ be a type where $s_{i}=(1, \ldots, 1)$ is of length $r_{i}$ for $1 \leq i \leq w$. Let $\mathfrak{A}=\left(A, G_{1}, \ldots, G_{w}\right)$ be a t-structure where $A=\{0, \ldots, n-1\}$ and $G_{i} \subseteq \mathcal{P}(A) \times \cdots \times \mathcal{P}(A)$. Denote by $\hat{\mathfrak{A}}=\left(B, P_{1}, \ldots, P_{w}\right)$ the following firstorder structure of vocabulary $\tau=\left\{P_{1}, \ldots, P_{w}\right\}$, where $P_{i}$ is a $r_{i}$-ary predicate, and

1. $B=\left\{0, \ldots, 2^{n}-1\right\}$,

2. $P_{i}=\left\{\left(j_{1}, \ldots, j_{r_{i}}\right) \in B^{r_{i}} \mid\left(J_{1}, \ldots, J_{r_{i}}\right) \in G_{i}\right\}$, where, for $1 \leq k \leq r_{i}, \operatorname{bin}\left(j_{k}\right)$ is given by $s_{0} \cdots s_{n-1}$, and $s_{l}=1 \Leftrightarrow l \in J_{k}$.

For a quantifier $\mathcal{Q}$ of type $t$, we denote by $\mathcal{Q}^{\star}$ the first-order quantifier of vocabulary $\tau$ defined by

$$
\mathcal{Q}^{\star}:=\{\hat{\mathfrak{A}}: \mathfrak{A} \in \mathcal{Q}\} .
$$

Note that the quantifier $\mathcal{Q}^{\star}$ has only structures in cardinalities of the form $2^{n}$ and that $\left|G_{i}\right|=\left|P_{i}\right|$ for $1 \leq i \leq w$. We are now ready for the main result of this article (see the Appendix for the proof).

Theorem 7. Let $\mathcal{Q}_{1}$ and $\mathcal{Q}_{2}$ be monadic quantifiers. Then $\mathcal{Q}_{1}$ is definable in $\operatorname{MSO}\left(\mathcal{Q}_{2},+\right)$ if and only if $\mathcal{Q}_{1}^{\star}$ is definable in $\operatorname{FO}\left(\mathcal{Q}_{2}^{\star},+, \times\right)$. 
Let us then discuss some corollaries of Theorem 7. We need the following definition.

Definition 7. Let $t=\left(s_{1}, \ldots, s_{w}\right)$ and $\tau$ be as in Definition 6. Let $\mathcal{Q}$ be a quantifier of type $t$. The quantifier $\mathcal{Q}$ is numerical if there is a relation $T \subseteq \mathbb{N}^{w}$ such that for all $t$-structures $\left(A, P_{1}, \ldots, P_{w}\right)$

$$
\left(A, P_{1}, \ldots, P_{w}\right) \in \mathcal{Q} \Leftrightarrow\left(\left|P_{1}\right|, \ldots,\left|P_{w}\right|\right) \in T .
$$

We denote $\mathcal{Q}$ by $\mathcal{Q}_{T}$ and by $Q_{T}$ the first-order numerical quantifier (defined analogously) of vocabulary $\tau$.

It is easy to see that, for a numerical $\mathcal{Q}_{T}$, the quantifier $\mathcal{Q}_{T}^{\star}$ (see Definition 6 ) is just the restriction of the corresponding first-order quantifier $Q_{T}$ to the cardinalities $2^{n}$ :

$$
\mathcal{Q}_{T}^{\star}=\left\{\left(A, P_{1}, \ldots, P_{w}\right) \in Q_{T}:|A|=2^{n} \text { for some } n \in \mathbb{N}\right\} .
$$

This observation allows us to show the following (see the Appendix for the proof):

Theorem 8. Let $\mathcal{Q}_{T}$ be a numerical quantifier and $k \in \mathbb{N}$. Then

1. $\mathcal{Q}_{T}$ is definable in $\mathrm{MSO}(+)$ iff $Q_{T}$ is definable in $\mathrm{FO}(+, \times)$.

2. $\mathcal{Q}_{T}$ is definable in $\mathrm{MSO}\left(\mathcal{D}_{k},+\right)$ iff $Q_{T}$ is definable in $\mathrm{FO}\left(\mathrm{D}_{k},+, \times\right)$.

3. $\mathcal{Q}_{T}$ is definable in $\mathrm{MSO}\left(\mathrm{Most}^{1},+\right)$ iff $Q_{T}$ is definable in $\mathrm{FO}(\mathrm{M},+, \times)$.

The following lemma can be now used.

Lemma 1. Let $S \subseteq \mathbb{N}, p$ a prime, and $q>1$ relatively prime to $p$. Then

1. $Q_{S}$ is definable in $\mathrm{FO}(+, \times)$ iff $S$ either finite or cofinite.

2. $\mathrm{D}_{q}$ is not definable in $\mathrm{FO}\left(\mathrm{D}_{p},+, \times\right)$.

Proof. The first claim follows from non-definability of the language PARITY in $\mathrm{FO}(+, \times)[28,29]$ (see Theorem 4.3 in [30]). The second claim goes back to [31].

By combining Theorem 8 and Lemma 1 we can show the following.

Corollary 1. Let $S \subseteq \mathbb{N}, p$ a prime, and $q>1$ relatively prime to $p$. Then

1. $\mathcal{Q}_{S}$ is definable in $\mathrm{MSO}(+)$ iff $S$ is either finite or cofinite.

2. $\mathcal{D}_{q}$ is not definable in $\operatorname{MSO}\left(\mathcal{D}_{p},+\right)$.

It is possible to replace $\mathrm{MSO}(+)$ by $\mathrm{SO}$ in Theorem 7. The idea is that, if $\mathcal{Q}_{1}$ is definable in $\mathrm{SO}\left(\mathcal{Q}_{2}\right)$, then in the defining formula, for some $k$, only relations of arity at most $k$ are quantified. We do not present this generalization in detail in this article but only consider the special case of the quantifier Most ${ }^{1}$.

Theorem 9. The quantifier Most ${ }^{1}$ is not definable in $\mathrm{SO}$. 
Proof. It suffices to show that Most ${ }^{1}$ is not definable in $\mathrm{FO}\left(\exists_{k}^{2}\right)$ for any $k$. Note also that $\left(\text { Most }^{1}\right)^{\star}$ is the restriction of $\mathrm{M}$ to the cardinalities $2^{n}$.

An analogous translation as in Theorem 7 can be used to show that definability of Most ${ }^{1}$ in SO implies that, for some $k$, the quantifier $\mathrm{M}$ is definable in $\mathrm{FO}(+, \times)$ over cardinalities $2^{n^{k}}$. Over these cardinalities, we could then express PARITY in the logic $\mathrm{FO}(+, \times)$. This contradicts the result of $[28,29]$.

In order to translate the quantifier $\exists_{k}^{2}$ to the $\operatorname{logic} \mathrm{FO}(+, \times)$, we redefine the structure $\hat{\mathfrak{A}}$ (see Definition 6 ) to have a domain of the form $\left\{0, \ldots, 2^{n^{k}}-1\right\}$. Now we can use the fact that there is a one-to-one correspondence between integers $m \in\left\{0, \ldots, 2^{n^{k}}-1\right\}$ and $k$-ary relations $R$ of $\{0, \ldots, n-1\}$. In other words, by using the lexicographic ordering on $k$-tuples, a relation $R$ can be encoded by a binary string of length $n^{k}$ corresponding to the binary representation of a unique integer $m<2^{n^{k}}$. It is straightforward to adjust the translation in the proof of Theorem 7 to this setting.

\section{Conclusion}

We have shown that definability of second-order generalized quantifiers can be reduced to definability of first-order generalized quantifiers. We have indicated a couple of corollaries to our characterization but surely there is more to be done here. Also, as discussed in connection to Theorem 9, it is possible to replace MSO in terms of SO and to prove a result analogous to Theorem 7 in this case. In particular, Theorem 9 solves the open problem proposed in [20], where we studied the collective meanings of natural language quantifiers. It suggests, as we argued in [20], that the type-shifting strategy [32] to define the meanings of natural language quantification might be too restricted in its computational power. It is likely that second-order logic is not enough to capture natural language semantics. Another interpretation would be that everyday language does not realize hard collective quantifiers (for sure they are marginal at best) due to their complexity.

\section{References}

1. Mostowski, A.: On a generalization of quantifiers. Fund. Math. 44 (1957) 12-36

2. Lindström, P.: First order predicate logic with generalized quantifiers. Theoria 32 (1966) 186-195

3. Väänänen, J.: Generalized quantifiers, an introduction. In: Generalized quantifiers and computation (Aix-en-Provence, 1997). Volume 1754 of Lecture Notes in Comput. Sci. Springer, Berlin (1999) 1-17

4. Ebbinghaus, H.D., Flum, J.: Finite model theory, 2nd edition. Perspectives in Mathematical Logic. Springer-Verlag (1999)

5. Peters, S., Westerståhl, D.: Quantifiers in Language and Logic. Clarendon Press, Oxford (2006)

6. Fagin, R.: Generalized first-order spectra and polynomial-time recognizable sets. In: Complexity of computation (Proc. SIAM-AMS Sympos. Appl. Math., New 
York, 1973). Amer. Math. Soc., Providence, R.I. (1974) 43-73. SIAM-AMS Proc., Vol. VII

7. Burtschick, H.J., Vollmer, H.: Lindström quantifiers and leaf language definability. Int. J. Found. Comput. Sci. 9(3) (1998) 277-294

8. Andersson, A.: On second-order generalized quantifiers and finite structures. Ann. Pure Appl. Logic 115(1-3) (2002) 1-32

9. Kontinen, J.: Definability of second order generalized quantifiers. PhD thesis, University of Helsinki (2005)

10. Kontinen, J.: Definability of second order generalized quantifiers. Arch. Math. Logic 49(3) (2010) 379-398

11. Kontinen, J.: The hierarchy theorem for second order generalized quantifiers. J. Symbolic Logic 71(1) (2006) 188-202

12. Bovet, D.P., Crescenzi, P., Silvestri, R.: A uniform approach to define complexity classes. Theor. Comput. Sci. 104(2) (1992) 263-283

13. Peichl, T., Vollmer, H.: Finite automata with generalized acceptance criteria. Discrete Mathematics and Theoretical Computer Science 4 (2001) 179-192

14. Galota, M., Vollmer, H.: A generalization of the Büchi-Elgot-Trakhtenbrottheorem. In: Computer Science Logic. Lecture Notes in Computer Science, Berlin Heidelberg, Springer Verlag (2001) 355-368

15. Büchi, J.R., Elgot, C.C.: Decision problems of weak second order arithmetics and finite automata, Part I. Notices of the American Mathematical Society 5 (1958) 834

16. Büchi, J.R.: On a decision method in restricted second-order arithmetic. In: Proceedings Logic, Methodology and Philosophy of Sciences 1960, Stanford, CA, Stanford University Press (1962)

17. Trakhtenbrot, B.A.: Finite automata and logic of monadic predicates. Doklady Akademii Nauk SSSR 140 (1961) 326-329 In Russian.

18. More, M., Olive, F.: Rudimentary languages and second-order logic. Math. Logic Quart. 43(3) (1997) 419-426

19. Torán, J.: Structural properties of the counting hierarchies. PhD thesis, Facultat d'Informatica de Barcelona, Barcelona, Spain (1988)

20. Kontinen, J., Szymanik, J.: A remark on collective quantification. Journal of Logic, Language and Information 17(2) (2008) 131-140

21. Szymanik, J.: Quantifiers in TIME and SPACE. Computational Complexity of Generalized Quantifiers in Natural Language. PhD thesis, Universiteit van Amsterdam (2009)

22. Immerman, N.: Descriptive complexity. Graduate Texts in Computer Science. Springer-Verlag, New York (1999)

23. Stockmeyer, L.J.: The polynomial-time hierarchy. Theor. Comput. Sci. 3(1) (1976) 1-22 (1977)

24. Parberry, I., Schnitger, G.: Parallel computation with threshold functions. J. Comput. System Sci. 36(3) (1988) 278-302

25. Barrington, D.A.M., Immerman, N., Straubing, H.: On uniformity within $\mathrm{NC}^{1}$. J. Comput. System Sci. 41(3) (1990) 274-306

26. Kontinen, J., Niemistö, H.: Extensions of MSO and the monadic counting hierarchy. Information and Computation 209(1) (2011) 1-19

27. Kontinen, J.: A logical characterization of the counting hierarchy. ACM Trans. Comput. Log. 10(1) (2009)

28. Furst, M.L., Saxe, J.B., Sipser, M.: Parity, circuits, and the polynomial-time hierarchy. Math. Systems Theory 17(1) (1984) 13-27 
29. Ajtai, M.: $\Sigma_{1}^{1}$-formulae on finite structures. Ann. Pure Appl. Logic 24(1) (1983) $1-48$

30. Barrington, D.A.M., Immerman, N., Lautemann, C., Schweikardt, N., Thérien, D.: First-order expressibility of languages with neutral letters or: The Crane Beach conjecture. J. Comput. System Sci. 70(2) (2005) 101-127

31. Smolensky, R.: Algebraic methods in the theory of lower bounds for boolean circuit complexity. In: STOC. (1987) 77-82

32. Winter, Y.: Flexibility principles in Boolean semantics. The MIT Press, London (2001)

33. Hesse, W., Allender, E., Barrington, D.A.M.: Uniform constant-depth threshold circuits for division and iterated multiplication. J. Comput. System Sci. 65(4) (2002) 695-716 Special issue on complexity, 2001 (Chicago, IL).

\section{Appendix}

Proof (of Theorem 7).

To simplify notation, we assume that the type of $\mathcal{Q}_{1}$ and $\mathcal{Q}_{2}$ is $((1,1))$ and $((1),(1))$, respectively.

Let us first assume that $\mathcal{Q}_{1}$ is definable in the $\operatorname{logic} \operatorname{MSO}\left(\mathcal{Q}_{2},+\right)$. Then there is a sentence $\phi \in \operatorname{MSO}\left(\mathcal{Q}_{2}, \mathcal{G},+\right)$ such that for all structures $(A,+, G)$

$$
(A,+, G) \models \phi \Leftrightarrow(A, G) \in \mathcal{Q}_{1} .
$$

We shall next show that there is a sentence $\phi^{*} \in \operatorname{FO}\left(\mathcal{Q}_{2}^{\star}+, \times\right)[\{P\}]$, where $P$ is binary, such that for all structures $\mathfrak{A}=(A, G)$ :

$$
(A,+, G) \models \phi \Leftrightarrow(B, P,<,+, \times) \models \phi^{*},
$$

where $(B, P)=\hat{\mathfrak{A}}$ (see Definition 6$)$. We define $\phi^{*}$ via the following translation:

$$
\begin{aligned}
x_{i}=x_{j} & \rightsquigarrow x_{i}=x_{j} \\
x_{i}+x_{j}=x_{k} & \rightsquigarrow x_{i}+x_{j}=x_{k} \\
Y_{i}\left(x_{j}\right) & \rightsquigarrow \operatorname{BIT}\left(y_{i}, n-\left(x_{j}+1\right)\right) \\
\mathcal{G} x_{i}, x_{j}\left(\psi_{1}\left(x_{i}\right), \psi_{2}\left(x_{j}\right)\right) & \rightsquigarrow \exists z_{1} \exists z_{2}\left(P ( z _ { 1 } , z _ { 2 } ) \wedge \bigwedge _ { 1 \leq i \leq 2 } \forall ( w < n ) \left(\psi_{i}^{*}(w)\right.\right. \\
\psi & \left.\left.\leftrightarrow \theta \operatorname{BIT}\left(z_{i}, n-(w+1)\right)\right)\right) \\
\neg \psi & \rightsquigarrow \neg \psi^{*} \wedge \theta^{*} \\
\exists x_{i} \psi & \rightsquigarrow \exists x_{i}\left(x_{i}<n \wedge \psi^{*}\left(x_{i}\right)\right) \\
\exists Y_{i} \psi & \rightsquigarrow \exists y_{i} \psi^{*} \\
\mathcal{Q}_{2} Y_{i}, Y_{j}\left(\psi\left(Y_{i}\right), \theta\left(Y_{j}\right)\right) & \rightsquigarrow \mathcal{Q}_{2}^{\star} y_{i}, y_{j}\left(\psi^{*}\left(y_{i}\right), \theta^{*}\left(y_{j}\right)\right)
\end{aligned}
$$

It is now straigthforward to show that for all formulas $\psi \in \operatorname{MSO}\left(\mathcal{Q}_{2}, \mathcal{G},+\right)$, structures $(A, G)$, and assignments $s$

$$
(A,+, G) \models_{s} \psi \Leftrightarrow(B, P,<,+, \times) \models_{s^{*}} \psi^{*},
$$


where the assignment $s^{*}$ is defined such that $s^{*}\left(x_{i}\right)=s\left(x_{i}\right)$ for all first-order variables $x_{i}$, and, if $s\left(Y_{i}\right)=D \subseteq\{0, \ldots, n-1\}$, then $s^{*}\left(y_{i}\right)$ is the unique $d<2^{n}$ whose binary representation is given by $s_{0} \cdots s_{n-1}$ where $s_{j}=1 \Longleftrightarrow j \in D$.

In the formula translation, we use the predicate $\mathrm{BIT}$, which is $\mathrm{FO}(+, \times)$ definable, to recover the set $D$ from the integer $d$. By the above translation, the sentence

$$
\exists n\left(|B|=2^{n} \wedge \phi^{*}\right)
$$

of the logic $\operatorname{FO}\left(\mathcal{Q}_{2}^{\star},+, \times\right)$ now defines the quantifier $\mathcal{Q}_{1}^{\star}$.

Let us then show the converse implication. Assume that $\phi \in \mathrm{FO}\left(\mathcal{Q}_{2}^{\star},+, \times\right)$ defines the quantifier $\mathcal{Q}_{1}^{\star}$. The idea is now to translate $\phi \in \mathrm{FO}\left(\mathcal{Q}_{2}^{\star},+, \times\right)$ to $\phi^{\prime} \in \operatorname{MSO}\left(\mathcal{Q}_{2}, \mathcal{G},+\right)$ such that for all $\mathfrak{A}=(A, G)$ :

$$
(A,+, G) \models \phi^{\prime} \Leftrightarrow(B, P,<,+, \times) \models \phi .
$$

Analogously to the first translation, we encode integers in the domain $B=$ $\left\{0, \ldots, 2^{n}-1\right\}$ in terms of subsets $X \subseteq\{0, \ldots, n-1\}$. We use the following formulas $X=Y, X<Y, X+Y=Z$, and $X \times Y=Z$ expressing arithmetic operations on binary numbers. The first three formulas are $\mathrm{FO}(+)$-expressible, and the fourth is expressible in the logic $\mathrm{FO}(\mathrm{M},+, \times) \leq \mathrm{MSO}(+)$ [33]. The translation $\phi \rightsquigarrow \phi^{\prime}$ is now defined as follows.

$$
\begin{aligned}
P\left(x_{i}, x_{j}\right) & \rightsquigarrow \mathcal{G} z_{1}, z_{2}\left(X_{i}\left(z_{1}\right), X_{j}\left(z_{2}\right)\right) \\
x_{i}=x_{j} & \rightsquigarrow X_{i}=X_{j} \\
x_{i}<x_{j} & \rightsquigarrow X_{i}<X_{j} \\
x_{i}+x_{j}=x_{k} & \rightsquigarrow X_{i}+X_{j}=X_{k} \\
x_{i} \times x_{j}=x_{k} & \rightsquigarrow X_{i} \times X_{j}=X_{k} \\
\psi \wedge \phi & \rightsquigarrow \psi^{\prime} \wedge \phi^{\prime} \\
\neg \psi & \rightsquigarrow \psi^{\prime} \\
\exists x_{i} \psi\left(x_{i}\right) & \rightsquigarrow \exists X_{i} \psi^{\prime}\left(X_{i}\right) \\
Q_{2}^{\star} x_{i}, x_{j}\left(\psi\left(x_{i}\right), \theta\left(x_{j}\right)\right) & \rightsquigarrow \mathcal{Q}_{2} X_{i}, X_{j}\left(\psi^{\prime}\left(X_{i}\right), \theta^{\prime}\left(X_{j}\right)\right)
\end{aligned}
$$

It is straightforward to show that this translation works as intended. In particular, it follows that the sentence $\phi^{\prime} \in \operatorname{MSO}\left(\mathcal{Q}_{2}, \mathcal{G},+\right)$ now defines the quantifier $\mathcal{Q}_{1}$.

Proof (of Theorem 8). The proof is based on the fact that each of the logics $\mathrm{FO}(<,+, \times), \mathrm{FO}\left(\mathrm{D}_{k},+, \times\right)$, and $\mathrm{FO}(\mathrm{M},+, \times)$ is closed under logical reductions. Suppose that $\mathcal{Q}_{T}$ is of type $t=\left(s_{1}, \ldots, s_{w}\right)$ and let $\tau$ denote the vocabulary of the corresponding first-order quantifier $Q_{T}$ (see Definition 6).

Let us consider claim 2. By Theorem 7 it suffices to show that the following are equivalent:

(a) $\mathcal{Q}_{T}^{\star}$ is definable in $\operatorname{FO}\left(\mathcal{D}_{k}^{\star},+, \times\right)$

(b) $Q_{T}$ is definable in $\mathrm{FO}\left(\mathrm{D}_{k},+, \times\right)$ 
Recall that the quantifiers $\mathcal{Q}_{T}^{\star}$ and $\mathcal{D}_{k}^{\star}$ are the restrictions of the quantifiers $Q_{T}$ and $\mathrm{D}_{k}$ to cardinalities of the form $2^{n}$, respectively. Let us first note that (a) is equivalent with

(c) $\mathcal{Q}_{T}^{\star}$ is definable in $\mathrm{FO}\left(\mathrm{D}_{k},+, \times\right)$.

First of all, since $\mathcal{D}_{k}^{\star}$ is easily definable in $\mathrm{FO}\left(\mathrm{D}_{k},+, \times\right)$ using the $\mathrm{FO}(+, \times)$ expressible predicate $x=2^{y}$, it follows that $(a) \Rightarrow(c)$. Assume then that (c) holds and let $\phi \in \operatorname{FO}\left(\mathrm{D}_{k},+, \times\right)$ define $\mathcal{Q}_{T}^{\star}$. Define a sentence $\psi$ as follows:

$$
\psi:=\exists n\left(|A|=2^{n} \wedge \phi\left(\mathrm{D}_{k} / \mathcal{D}_{k}^{\star}\right)\right) .
$$

Since the quantifier $\mathcal{Q}_{T}^{\star}$ contains structures only in cardinalities of the form $2^{n}$ it is easy to see that $\psi \in \operatorname{FO}\left(\mathcal{D}_{k}^{\star},+, \times\right)$ also defines $\mathcal{Q}_{T}^{\star}$.

It now suffices to show that (b) and (c) are equivalent. Note first that $(b) \Rightarrow$ (c) can be easily proved using the predicate $x=2^{y}$. We will show $(c) \Rightarrow(b)$. Here we use the fact that the logic $\mathrm{FO}\left(\mathrm{D}_{k},+, \times\right)$ is closed under logical reductions. We will define $Q_{T}$ (over all cardinalities) with the help of the quantifier $\mathcal{Q}_{T}^{\star}$. Let $\mathfrak{A}$ be a structure. If $|A|=2^{n}$ for some $n \in \mathbb{N}$, then $\mathfrak{A} \in Q_{T}$ can be expressed in terms of the quantifier $\mathcal{Q}_{T}^{\star}$. Note that even if $|A|$ is not a power of two, it holds that the least $m$ such that $|A| \leq 2^{m}$ satisfies $2^{m} \leq|A|^{2}$.

We will now sketch how the quantifier $Q_{T}$ can be defined in terms of $\mathcal{Q}_{T}^{\star}$. Assume $\phi \in \mathrm{FO}\left(\mathrm{D}_{k},+, \times\right)$ is a sentence defining $\mathcal{Q}_{T}^{\star}$. Let $\mathfrak{A}=\left(A, P_{1}, \ldots, P_{w}\right)$ be a $\tau$-structure, where $A=\{0, \ldots, n-1\}$. We use the following facts:

1. There is a $\mathrm{FO}(<,+, \times)$-definable query $I$ that maps $\mathfrak{A}$ to the structure $I(\mathfrak{A})$ which is isomorphic to

$$
\left(\left\{0, \ldots, 2^{m}-1\right\}, P_{1}, \ldots, P_{w},<,+, \times\right),
$$

where $2^{m}$ is the least power of two satisfying $n \leq 2^{m}$.

2. There is a sentence $\psi \in \mathrm{FO}\left(\mathrm{D}_{k},+, \times\right)$ such that for all $\mathfrak{A}$ :

$$
\mathfrak{A} \models \psi \Leftrightarrow I(\mathfrak{A}) \models \phi .
$$

Since $Q_{T}$ is numerical, the sentence $\psi$ now defines $Q_{T}$. The query $I$ is easily definable in $\mathrm{FO}(<,+, \times)$; the domain of $I(\mathfrak{A})$ is defined as $\left\{(i, j) \in A^{2} \mid i n+j<\right.$ $\left.2^{m}\right\}$ (see [22] for more on first-order queries). The sentence $\psi$ is constructed inductively (see e.g., Section $3.2[22]$ ) using, in particular, the fact that the second vectorization $\mathrm{D}_{k}^{2}$ of $\mathrm{D}_{k}$ can be expressed in $\mathrm{FO}\left(\mathrm{D}_{k},+, \times\right)$.

The claims 1 are 3 are proved analogously. For claim 3 we use the facts that $\left(\mathrm{Most}^{1}\right)^{\star}$ is the restriction of $\mathrm{M}$ to the cardinalities $2^{n}$ and that the second vectorization $\mathrm{M}^{2}$ of $\mathrm{M}$ is definable in $\mathrm{FO}(\mathrm{M},+, \times)$ (see [25]). 